\title{
Prague Hereditary Hypercholesterolemic (PHHC) Rat - a Model of Polygenic Hypercholesterolemia
}

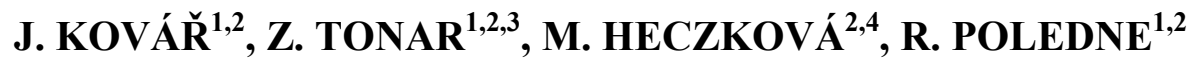 \\ ${ }^{1}$ Center for Cardiovascular Research, Prague, ${ }^{2}$ Laboratory for Atherosclerosis Research, Institute \\ for Clinical and Experimental Medicine, Prague, ${ }^{3}$ Department of Histology and Embryology, \\ Faculty of Medicine in Pilsen, Charles University in Prague, Pilsen, ${ }^{4}$ Department of Medical \\ Biology, Faculty of Science, University of South Bohemia, České Budějovice, Czech Republic
}

Received July 11, 2009

Accepted October 30, 2009

\section{Summary}

Prague hereditary hypercholesterolemic (PHHC) rat - rat strain crossbred from Wistar rats - is a model of hypercholesterolemia induced by dietary cholesterol. Importantly, no bile salts and/or antithyroid drugs need to be added to the diet together with cholesterol to induce hypercholesterolemia. PHHC rats have only modestly increased cholesterolemia when fed a standard chow and develop hypercholesterolemia exceeding $5 \mathrm{mmol} / \mathrm{l}$ on $2 \%$ cholesterol diet. Most of the cholesterol in hypercholesterolemic PHHC rats is found in VLDL that become enriched with cholesterol (VLDL-C/VLDL-TG ratio > 1.0). Concurrently, both IDL and LDL concentrations rise without any increase in HDL. PHHC rats do not markedly differ from Wistar rats in the activities of enzymes involved in intravascular remodelation of lipoproteins (lipoprotein and hepatic lipases and lecithin:cholesterol acyltransferase), LDL catabolism, cholesterol turnover rate and absorption of dietary cholesterol. The feeding rats with cholesterol diet results in development of fatty liver in spite of suppression of cholesterol synthesis. However, even though cholesterolemia in $\mathrm{PHHC}$ rats is comparable to human hypercholesterolemia, the PHHC rats do not develop atherosclerosis even after 6 months on $2 \%$ cholesterol diet. Importantly, the crossbreeding experiments documented that hypercholesterolemia of $\mathrm{PHHC}$ rats is polygenic. To identify the genes that may be involved in pathogenesis of hypercholesterolemia in this strain, the studies of microarray gene expression in the liver of $\mathrm{PHHC}$ rats are currently in progress.

\section{Key words}

Rat $\bullet$ Hypercholesterolemia • Genetics • Cholesterol metabolism

\section{Corresponding author}

J. Kovář, Institute for Clinical and Experimental Medicine, Laboratory for Atherosclerosis Research, Vídeňská 1958/9, 14021 Prague 4, Czech Republic. E-mail: jan.kovar@medicon.cz

\section{Introduction}

In spite of remarkable progress in therapy of hypercholesterolemia in recent years, cardiovascular disease remains to be a leading cause of mortality worldwide. Although our understanding of pathogenesis of hypercholesterolemia made great progress and we have deep insight of some monogenic disorders, most of the patients have a hypercholesterolemia that is polygenic. The genes that are involved in its pathogenesis have not been fully characterized yet. Their identification in humans is a quite complex problem and it would be very helpful to have appropriate experimental models. It should be stressed that the knock-out and/or transgene animals used up to now are used to confirm the role of already identified candidate genes but cannot contribute to the discovery of the other genes involved in the pathogenesis of hypercholesterolemia.

One of the animals often used in research as a model animal - rat - does not have a prominent role in lipoprotein metabolism and atherosclerosis research. Rats 
have very low serum cholesterol (usually under $2 \mathrm{mmol} / \mathrm{l}$ ) and they belong to "HDL-mammals" - most of their cholesterol is carried out in high density lipoproteins (HDL) (Chapman 1986). Moreover, rats are very resistant to dietary cholesterol - to produce hypercholesterolemia, cholic acid and/or drugs affecting thyroid function has to be added to cholesterol diet (Mahley and Holcombe 1977, DeLamatre and Roheim 1981, Cole et al. 1984). Although exact mechanism behind cholic acid action has not been unambiguously identified yet, it can inhibit bile acid synthesis in the liver and thus block the major pathway of cholesterol catabolism. Alternatively, the hepatotoxic effects of high concentration of cholic acid may also play a role. Even if a rat model responsive to dietary cholesterol (RICO rats) was obtained, the hypercholesterolemia in this model is rather mild and do not exceed $5 \mathrm{mmol} / \mathrm{l}$, concentration that is considered normal in humans (Ouguerram et al. 1996). Moreover, hypercholesterolemia is due to an increase in HDLcholesterol to a great extent, which limits the use of this model for study of the role of hypercholesterolemia in pathogenesis of atherosclerosis.

\section{Development of the model and mode of inheritance}

In order to establish a model of hypercholesterolemia in rats, the selective inbreeding of the Wistar rats that were most responsive to dietary cholesterol (without any addition of cholic acid and/or thyreotoxic substances) was carried out. Briefly, five pairs of rats with highest basal cholesterolemia were selected from 100 rats for parent generation. In each generation, cholesterol was then measured in the rats on a standard chow and the animals were then shifted to $2 \%$ cholesterol diet (cholesterol dissolved in $5 \%$ beef tallow) for 2 weeks and cholesterol was determined again. The rats with maximal increase in cholesterolemia were then selected for further breeding. Although there were no dramatic changes in the baseline cholesterolemia $(\sim 2.0 \mathrm{mmol} / \mathrm{l})$ and even in dietary cholesterol stimulated cholesterolemia (2-3 mmol/l in males and 3-4 mmol/l in females) during first 8 generations, there was a dramatic shift of dietary induced cholesterolemia to concentration $\sim 10 \mathrm{mmol} / \mathrm{l}$ after the $9^{\text {th }}$ generation likely due to a gene recombination (Poledne 1986).

At the same time, the other line of animals that were not responsive to dietary cholesterol was selected by breeding animals unresponsive to dietary cholesterol.
However, this line was lost after thirteen generations due to the low fertility.

To assess the mode of transmission of hypercholesterolemia, the PHHC animals were crossbred with the animals of control line not responsive to dietary cholesterol. The offspring $\left(\mathrm{F}_{1}\right)$ displayed the dietary induced hypercholesterolemia that was at the mid level between cholesterol concentrations of both parental strains. The standard deviation of cholesterolemia of $F_{1}$ generation did not differ from that of their parents. If the dietary induced cholesterolemia would be a monogenic trait, it could be predicted that distribution of cholesterolemia in offspring of $F_{1}$ animals ( $F_{2}$ generation) should have three discrete peaks corresponding to cholesterolemia of both parental strains and $F_{1}$ generation in the ratio $1: 1: 2$, respectively. That was not a case. The cholesterolemia distribution in $\mathrm{F}_{2}$ generation displayed a single peak at concentration corresponding to that of $F_{1}$ generation and very high standard deviation. That strongly suggest that hypercholesterolemia in PHHC rats is polygenic (Reysserová and Poledne, unpublished data).

\section{Lipoproteins in PHHC rat}

When fed a standard chow, PHHC rats display only moderately increased cholesterolemia in comparison to control Wistar rats. There is no obvious difference in the lipoprotein profile between both strains - the major part of cholesterol is carried out in HDL in both strains (Fig. 1). When fed $1 \%$ or $2 \%$ cholesterol diet (without any addition of cholic acid), serum cholesterol concentration goes a little bit up in Wistar rats and rises severalfold in PHHC rats. Females of PHHC rats seem to be more sensitive to dietary cholesterol than males. Importantly, most of the cholesterol in PHHC rats on cholesterol diet is carried out in very low density lipoproteins (VLDL) and intermediate density lipoproteins (IDL). Cholesterol concentration in low density lipoproteins (LDL) goes also up, although it is likely that ultracentrifugally isolated LDL are contaminated by large apo E-enriched $\mathrm{HDL}_{\mathrm{c}}$ particles as described by others (Mahley and Holcombe 1977). HDL ( $\mathrm{d}>1.063 \mathrm{~g} / \mathrm{ml}$ ) concentration is rather slightly lower in cholesterol-fed PHHC rats. The serum triglyceride (TG) concentration is not affected, therefore VLDL become cholesterol-rich (VLDLC/VLDL-TG ratio usually $>1.0$ ) and the same applies for IDL that carry more cholesterol than TG. 


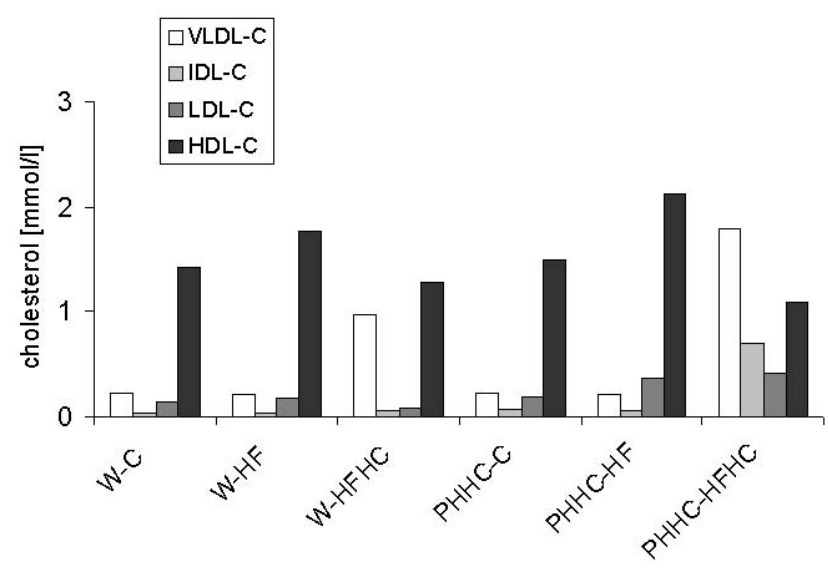

Fig. 1. Cholesterol concentration in ultracentrifugally isolated lipoproteins in PHHC and Wistar rats. The sequential ultracentrifugation (Havel et al. 1955) was carried out on pools of sera obtained from experiment described in Table 1.

The high VLDL-C/VLDL-TG ratio and high concentration of IDL indicate that lipoprotein profile of PHHC rats is similar to that of dysbetalipoproteinemia (hyperlipoproteinemia type III) in humans. Dysbetalipoproteinemia is a disorder characterized by the inability of apolipoprotein $\mathrm{E}$ interact with cellular receptors resulting in accumulation of chylomicron remnants and remnant VLDL. Therefore we addressed the question whether defective apolipoprotein $\mathrm{E}$ is involved in pathogenesis of hyperlipoproteinemia in PHHC rats. Apolipoprotein $\mathrm{E}$ gene (Apoe) in $\mathrm{PHHC}$ and Wistar rats was sequenced and mutation G24C was found in the first of four Apoe exons (data not shown). However, the first exon of the gene is not translated and the mutation thus should not affect the primary structure of the protein. Importantly, when VLDL of rats are isolated and their apolipoproteins separated by polyacrylamide gradient gel electrophoresis (Bergeron et al. 1996), apolipoprotein E can be detected in VLDL of both PHHC and Wistar rats at comparable level. This suggest that the G24C mutation does not seem to have any effect on gene transcription and/or translation and is unlikely to explain the pathogenesis of hyperlipo-proteinemia in PHHC rats.

\section{Intravascular lipoprotein metabolism in PHHC rat}

There are no major differences between PHHC and Wistar rats in lipoprotein lipase activity. Hepatic lipase activity was found to be modestly increased $(\sim 30 \%)$ in PHHC rats in comparison to Wistar rats on chow and was suppressed by $20 \%$ after 2 weeks on cholesterol diet in both strains (Bařinka 1995). No pronounced differences between both strains were found in lecithin:cholesterol acyltransferase activity measured using both method of autologous and homologous substrate (Befekadu et al. 1992). Therefore it does not seem that these key enzymes of intravascular lipoprotein metabolism are involved in the development of hypercholesterolemia in PHHC rats.

When ${ }^{125}$ I-labelled LDL were i.v. injected to both PHHC and Wistar rats on cholesterol diet, no difference in the rate of their disappearance from circulation could be observed suggestive of the fact that there is no difference in the feedback regulation of LDL receptor by cholesterol (Poledne 1986). The total body turnover time is prolonged in PHHC rat compared to Wistar rat and there is no difference in the rate of absorption of dietary cholesterol between both strains (Poledne 1986).

\section{Metabolism of cholesterol in the liver}

The cholesterol starts to accumulate in the liver of PHHC rats already after one day feeding with cholesterol. The accumulation progresses further with the time (Befekadu et al. 1992) and is accompanied by triglyceride accumulation finally developing into fatty liver. Interestingly, the presence of cholesterol in the diet is necessary for $\mathrm{TG}$ accumulation in the liver, the $5 \%$ dietary fat alone has no such effect (Table 1). The synthesis of cholesterol from ${ }^{3} \mathrm{H}_{2} \mathrm{O}$ in the liver measured as described by Turley et al. (1981) is down-regulated by the accumulated cholesterol, although the same level of suppression is achieved at the approximately twofold cholesterol concentration in the liver in PHHC rats than in Wistar rats (Poledne 1986). Recent data suggested that PHHC rats may not be able to respond to the dietary cholesterol by an increase in cholesterol $7 \alpha$-hydroxylase (CYP7A1) gene expression (Hubáček et al. 2008) and, therefore, they are unable to metabolize excessive cholesterol to bile acids.

\section{Atherosclerosis and PHHC rat}

Although PHHC rat seems to be a very convenient model to study the mechanisms involved in the regulation of cholesterolemia that is more close to humans than the other rat models of hyperchole-sterolemia, we found that even rats kept for 6 months on $2 \%$ cholesterol diet (with cholesterolemia $18 \pm 10 \mathrm{mmol} / \mathrm{l}$ ) were completely resistant to the development of atherosclerosis. No 
Table 1. Cholesterol and triglyceride in serum and liver of Wistar and PHHC rats.

\begin{tabular}{|c|c|c|c|c|c|c|}
\hline \multirow[b]{3}{*}{ n } & \multicolumn{3}{|c|}{ Wistar } & \multicolumn{3}{|c|}{ PHHC } \\
\hline & $\mathrm{C}$ & HF & HFHC & $\mathrm{C}$ & HF & HFHC \\
\hline & 8 & 8 & 8 & 6 & 6 & 7 \\
\hline $\begin{array}{l}\text { Serum } \\
\text { cholesterol } \\
(\mathrm{mmol} / \mathrm{l})\end{array}$ & $1.68^{\mathrm{b}} \pm 0.19$ & $1.98^{\mathrm{a}, \mathrm{b}} \pm 0.26$ & $2.34^{\mathrm{a}} \pm 0.37$ & $2.48^{\mathrm{b}^{*}} \pm 0.21$ & $2.71^{b^{*}} \pm 0.26$ & $4.24^{\mathrm{a}^{*}} \pm 0.24$ \\
\hline $\begin{array}{l}\text { Serum TG } \\
(\mathrm{mmol} / \mathrm{l})\end{array}$ & $1.22 \pm 0.18$ & $1.24 \pm 0.42$ & $1.79 \pm 0.39$ & $0.92 \pm 0.21$ & $0.95 \pm 0.38$ & $1.42 \pm 0.47$ \\
\hline $\begin{array}{l}\text { Liver cholesterol } \\
(\mu \mathrm{mol} / \mathrm{l})\end{array}$ & $6.09^{\mathrm{b}} \pm 1.31$ & $5.83^{\mathrm{b}} \pm 0.39$ & $24.30^{\mathrm{a}} \pm 4.49$ & $5.98^{\mathrm{b}} \pm 0.66$ & $5.58^{\mathrm{b}} \pm 1.63$ & $27.55^{\mathrm{a}} \pm 13.06$ \\
\hline $\begin{array}{l}\text { Liver TG } \\
(\mu \mathrm{mol} / \mathrm{l})\end{array}$ & $3.60^{b} \pm 0.51$ & $4.66^{\mathrm{b}} \pm 1.11$ & $14.83^{\mathrm{a}} \pm 2.57$ & $3.12^{\mathrm{b}} \pm 0.70$ & $4.1^{\mathrm{b}} \pm 0.77$ & $14.54^{\mathrm{a}} \pm 3.64$ \\
\hline
\end{tabular}

Wistar and PHHC rats (males 270-450 g of weight) were fed chow (C), high-fat diet (HF) (5\% palm oil), and high-fat high-cholesterol diet (HFHC) (1\% cholesterol; $5 \%$ palm oil) for three weeks. Cholesterol and triglycerides were determined at the end of experiment using commercially available enzymatic kits in sera and in Folch extracts of liver aliquots. ${ }^{*} \ldots \mathrm{p}<0.01$ Wistar vs PHHC rats on the same diet, $a, b$... the same letters are assigned to the diet treatments within the same strain that do not differ $(p>0.05)$.
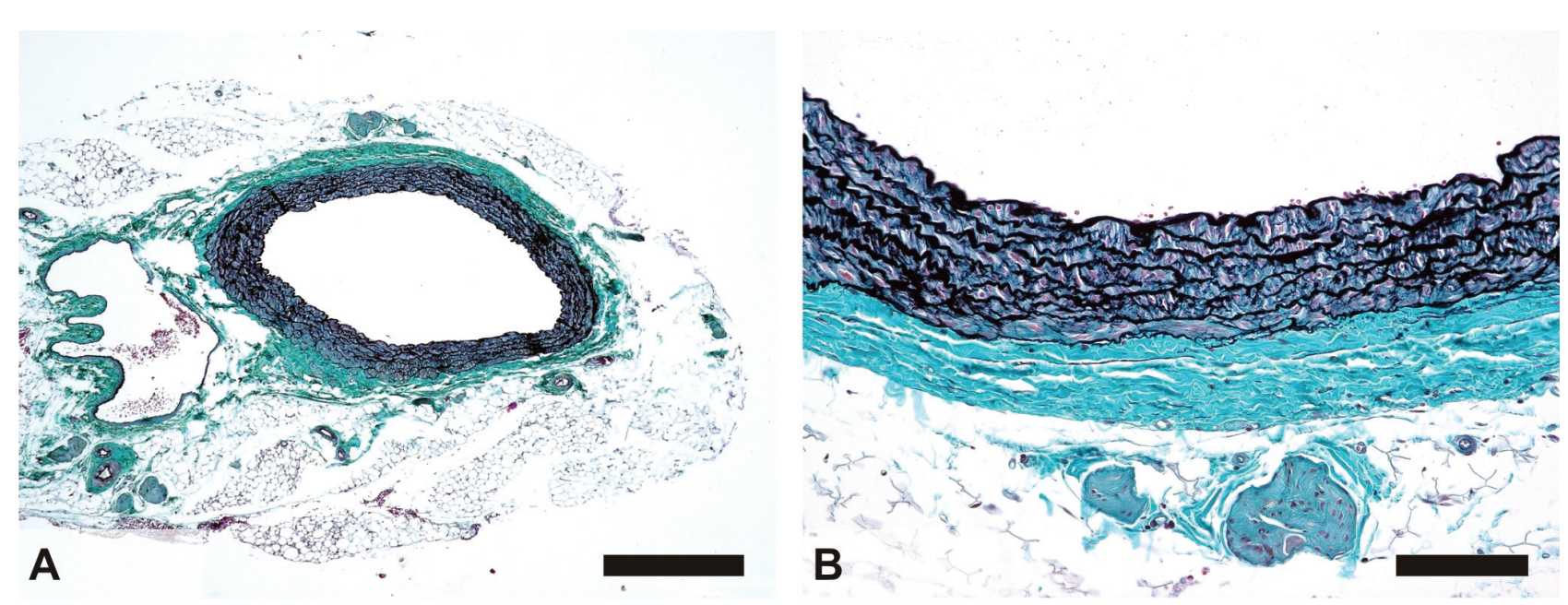

Fig. 2. Histology of ascending thoracic aorta of a PHHC rat on cholesterol diet. The findings of a rat randomly selected from the group fed $2 \%$ cholesterol diet for 6 months is demonstrated with a $5 \mu \mathrm{m}$ thick cross section (A) stained with Verhoeff's hematoxylin and green trichrome (Kočová 1970). The aorta was apparently free of any atherosclerotic lesion. Even in a high-power micrograph (B), there was no thickening of the subendothelial connective tissue in the tunica intima. The tunica media was clearly delimited with the internal and external elastic laminae as it consisted of regularly repeating lamellar units of elastin (stained black), collagen fibers (green), and smooth muscle cells (reddish or brown). Scale bar of $600 \mu \mathrm{m}$ (A) and $120 \mu \mathrm{m}$ (B). No differences were observed between PHHC rats fed $2 \%$ cholesterol diet and those fed chow.

macroscopic lesions were observed in aorta immediately after killing these animals and no differences in histology of ascending thoracic aorta between PHHC rats on cholesterol diet and those of the same age kept on chow were found (Fig. 2).

\section{Future directions}

The PHHC rats represent a model of polygenic hypercholesterolemia of unknown etiology. The exact mechanism leading to development of hypercholesterolemia still needs to be determined. Especially, it remains to be clarified whether cholesterol-enriched VLDL are produced directly in the liver or whether these particles originate in circulation from normal VLDL. As summarized above, except of differences in CYP7A1 gene expression, no pronounced differences between PHHC and Wistar rats could be identified and due to a 
polygenic nature of the model it can be anticipated that some new genes, which can play a role in regulation of cholesterolemia and/or cholesterol metabolism, may be uncovered in PHHC rats. To examine such a possibility, the studies of microarray gene expression in the liver of $\mathrm{PHHC}$ rats are currently in progress.

\section{Conflict of Interest}

There is no conflict of interest.

\section{Acknowledgements}

The project was supported by grant NR 9401-3 from IGA MH CR.

\section{References}

BǍ̆INKA C: Lipoprotein and Hepatic Lipase in Model of Experimental Hyperlipoproteinemia (in Czech). MSc Theses. Prague, Department of Biochemistry, Faculty of Sciences, Charles University Prague, 1995.

BEFEKADU G, KOVÁŘ J, POLEDNE R: High sensitivity of PHHC rat to dietary cholesterol. Physiol Res 41: 263266, 1992.

BERGERON N, KOTITE L, HAVEL RJ: Simultaneous quantification of apolipoproteins B-100, B-48, and E separated by SDS-PAGE. Methods Enzymol 263: 82-94, 1996.

CHAPMAN MJ: Comparative analysis of mammalian plasma lipoproteins. Methods Enzymol 128: 70-143, 1986.

COLE TG, KUISK I, PATSCH W, SCHONFELD G: Effects of high cholesterol diets on rat plasma lipoproteins and lipoprotein-cell interactions. J Lipid Res 25: 593-603, 1984.

DELAMATRE JG, ROHEIM PS: Effect of cholesterol feeding on apo B and apo E concentrations and distributions in euthyroid and hypothyroid rats. J Lipid Res 22: 297-306, 1981.

HAVEL RJ, EDER H, BRAGDON J: The distribution and chemical composition of ultracentrifugally separated lipoproteins in human serum. J Clin Invest 34: 1345-1353, 1955.

HUBÁČEK JA, BOBKOVÁ D, BOHUSLAVOVÁ R, POLEDNE R: Differences in expression of cholesterol 7 $\alpha$-hydroxylase between PHHC and Wistar rats. Folia Biol (Praha) 54: 18-23, 2008.

KOČOVÁ J: Overall staining of connective tissue and the muscular layer of vessels. Folia Morphol (Praha) 3: 293295, 1970.

MAHLEY RW, HOLCOMBE KS: Alterations of the plasma lipoproteins and apoproteins following cholesterol feeding in the rat. J Lipid Res 18: 314-324, 1977.

OUGUERRAM K, MAGOT T, LUTTON C: Metabolism of plasma lipoproteins in the genetically hypercholesterolemic rat (RICO). Metabolism 45: 4-11, 1996.

POLEDNE R: Effect of diet on cholesterol metabolism in the Prague hereditary hypercholesterolaemic rat. In: Nutritional Effects on Cholesterol Metabolism. AC BEYNEN (ed), Transmondial, Voorthuizen, 1986, pp 91-97.

TURLEY SD, ANDERSEN JM, DIETSCHY JM: Rates of sterol synthesis and uptake in the major organs of the rat in vivo. J Lipid Res 22: 551-569, 1981. 